\title{
Il Battistero di Firenze nella storiografia medicea tra Cosimo I e Francesco I
}

\author{
Eliana Carrara and Emanuela Ferretti*
}

\subsection{Borghini, Vasari e il Romanico fiorentino: il genius loci e l'Antico}

'Avvertiscasi se il modo di fare le facciate de' tempii et le [...] incrostature delle mura di varii marmi et colori, come è la chiesa di S. Giovanni et di S. Maria del Fiore et la facciata di $\mathrm{S}$. Miniato et della Badia di Fiesole è cosa antica, cioè del buon tempo de' Romani, o pure fu trovato de' Gotti et Longobardi. Non perché io non sappia che queste di S. Giovanni et di S. Miniato son moderne, et sassi a che tempo et da chi furon fatte, ma lo dico se fusse a proposito servirsene in discorrendo come in tempi diversi è variato il modo et la forma del fabbricare. Hammi messo questo sospetto le parole di S. Gregorio Turronense c. 288 variavit marmore. Et bisognando se ne potrà scrivere a Roma; ma quanto a' pavimenti che si facessero anchor ne' buoni tempi di musaico di varie pietre, credo che ne sia assai buona certezza.' ${ }^{1}$

Vincenzio Borghini (1515-1580) scrive questo passo alla metà degli anni sessanta del Cinquecento, mentre era in corso da parte di Giorgio Vasari (15111574) la riscritture delle Vite, pubblicate poi dai Giunti nel 1568. L'edizione dei Giunti, come è noto, è stata molto ampliata rispetto a quella apparsa presso Torrentino nel 1550, a cui Vincenzio Borghini collaborò attivamente con indicazioni e suggerimenti che Vasari tenne nel dovuto conto. Borghini, dotto

* Benché il saggio nasca da un'articolata riflessione comune, il primo paragrafo si deve a Emanuela Ferretti, mentre il secondo a Eliana Carrara. Criteri di trascrizione. Citeremo i testi cinquecenteschi secondo i seguenti criteri: è stata distinta $u$ da $v$; si è reso $j$ con $i$; sono introdotti accenti, apostrofo e segni d'interpunzione secondo l'uso odierno, così come la divisione delle parole e l'uso delle maiuscole (ma sono state conservate alcune occorrenze dell'impiego delle lettere maiuscole per rispettare una consuetudine del linguaggio di corte: ad es. Duca, Signore); sono state sciolte tutte le abbreviazioni senza darne conto (tranne titoli onorifici quali V.A. = Vostra Altezza). Fra parentesi quadre, infine, sarà posto ogni nostro intervento di emendazione o integrazione. Con una sbarretta trasversale si indica l'avvenuto cambio della carta.

1 BAV, Chigiano, L V 178, c. 5or. Cfr. E. Carrara, 'Doni, Vasari, Borghini e la tecnica del mosaico', in: G. Bertoli, R. Drusi (eds.), Fra lo spedale e il principe. Vincenzio Borghini: filologia e invenzione nella Firenze di Cosimo I, Padova (2005), 79-93: 91-92. 
monaco benedettino, è una figura chiave della corte di Cosimo I de' Medici, oltre a essere stato uno dei punti di riferimento della vita culturale fiorentina della seconda metà del secolo. ${ }^{2}$

Il passo focalizza due tematiche, ovvero il 'modo' e la 'forma del fabbricare' che metteva, agli occhi di Borghini, sullo stesso piano, e in un succedersi diretto - fra gli altri - i rivestimenti esterni del Battistero e della facciata della chiesa di S. Miniato al Monte. ${ }^{3}$ Tali esempi furono poi presentati da Vasari in stretta contiguità alle architetture fiorentine di Leon Battista Alberti, come la facciata di S. Maria Novella (fig. 10.1), in cui i prodigi tecnici dell'antichità trovarono nuova attuazione. Scrive infatti Vasari:

$\mathrm{A}^{\prime}$ dì nostri non si è mai condotto pietre di questa sorte a perfezzione alcuna per avere gli artefici nostri perduto il modo del temperare i ferri e così gli altri stormenti da condurre. [...] E se bene si sono in diversi tempi provati molti belli ingegni per trovare il modo di lavorarlo che usarono gli antichi, tutto è stato invano; e Leon Battista Alberti, il quale fu il primo che cominciasse a far pruova di lavorarlo, non però in cose di molto momento; [...] [se ne] servì nondimeno di maniera che fece fare nella soglia della porta principale di Santa Maria Novella di Fiorenza le diciotto lettere antiche che, assai grandi e ben misurate, si veggono dalla parte dinanzi in un pezzo di porfido. ${ }^{4}$

Nel testo vasariano possiamo enucleare un concetto basilare: la continuità nel contesto fiorentino fra mondo antico, mondo medioevale e la rinascita quattrocentesca dell'arte e dell'architettura attraverso l'ars come techne. Tutto ciò viene esemplificato, sempre in riferimento al brano vasariano sopra citato, grazie al confronto fra la facciata di San Miniato e quella di Santa Maria Novella. La facciata di quest'ultima basilica, a propria volta, è uno degli esempi più rilevanti per Vasari della continuità e contiguità fra le capacità tecniche e gli esiti

2 Si veda da ultima, E. Carrara, "Così feci il fangotto et portai tutti e libri soprascritti". Postille per un resoconto di vent'anni di studi borghiniani', in: E. Carrara, M. Fubini Leuzzi (eds.), I Ricordi di Vincenzio Borghini, Roma 2018, 1-11. Su Vasari si veda: B. Agosti, Giorgio Vasari. Luoghi e tempi delle Vite, Milano 2016.

3 E. Carrara, 'Tra fonti e immagini. La polemica sul Battistero fiorentino negli scritti di don Vincenzo Borghini', in: U. Rozzo, M. Gabriele (eds.), Storia per parole e per immagini, atti del convegno (Cividale del Friuli, 2003), Udine 2006, 193-211; F.P. Di Teodoro, "Marmorea Templa": Firenze, identità romana e tutela identitaria', in: H. Burns, M. Mussolini (eds.), Architettura e identità locali, II, Firenze 2013, 449-469.

4 Giorgio Vasari, Le vite de' più eccellenti pittori, scultori e architettori nelle redazioni del 1550 e 1568, (eds. R. Bettarini and P. Barocchi), Firenze 1966-1987, I, 33-34. 


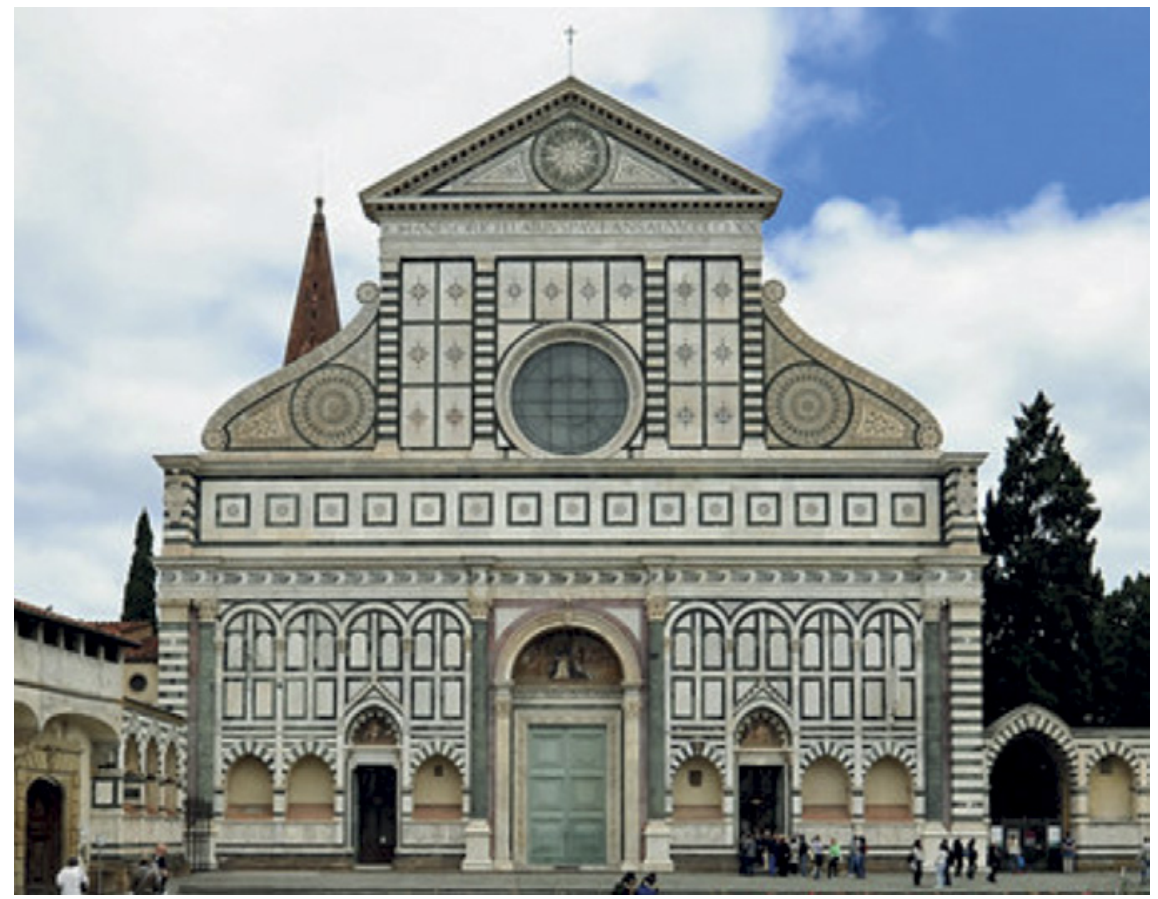

FIGURE 10.1 Santa Maria Novella, Firenze

FOTO: E. FERRETTI

stilistici degli antichi e dei moderni, che si palesa attraverso le caratteristiche realizzazioni del Romanico fiorentino. ${ }^{5}$

Nella lettura vasariana, infatti, le chiese romaniche di Firenze rappresentano l'anello di congiunzione tra 'la buona arte degli antichi' e l'altrettanto e ancora più valente arte degli architetti moderni:

San Giovanni [l'architettura del quale] è dagl' architetti moderni come cosa singolare lodata, e meritamente, perciò che ell'ha mostrato il buono che già aveva in sé quell'arte a Filippo di ser Brunelesco, a Donatello et agl'altri maestri di que' tempi, i quali impararono l'arte col mezzo di quell'opera e della chiesa di S. Apostolo di Firenze. ${ }^{6}$

5 E. Carrara, E. Ferretti, "Il bellissimo bianco" della Sacrestia Nuova: Michelangelo, Vasari, Borghini e la tradizione fiorentina come nuova identità medicea', Opus Incertum, n.s., II (2016), 58-73: 73 .

6 Vasari, Le vite, op. cit. (nota 4), II, 74. 


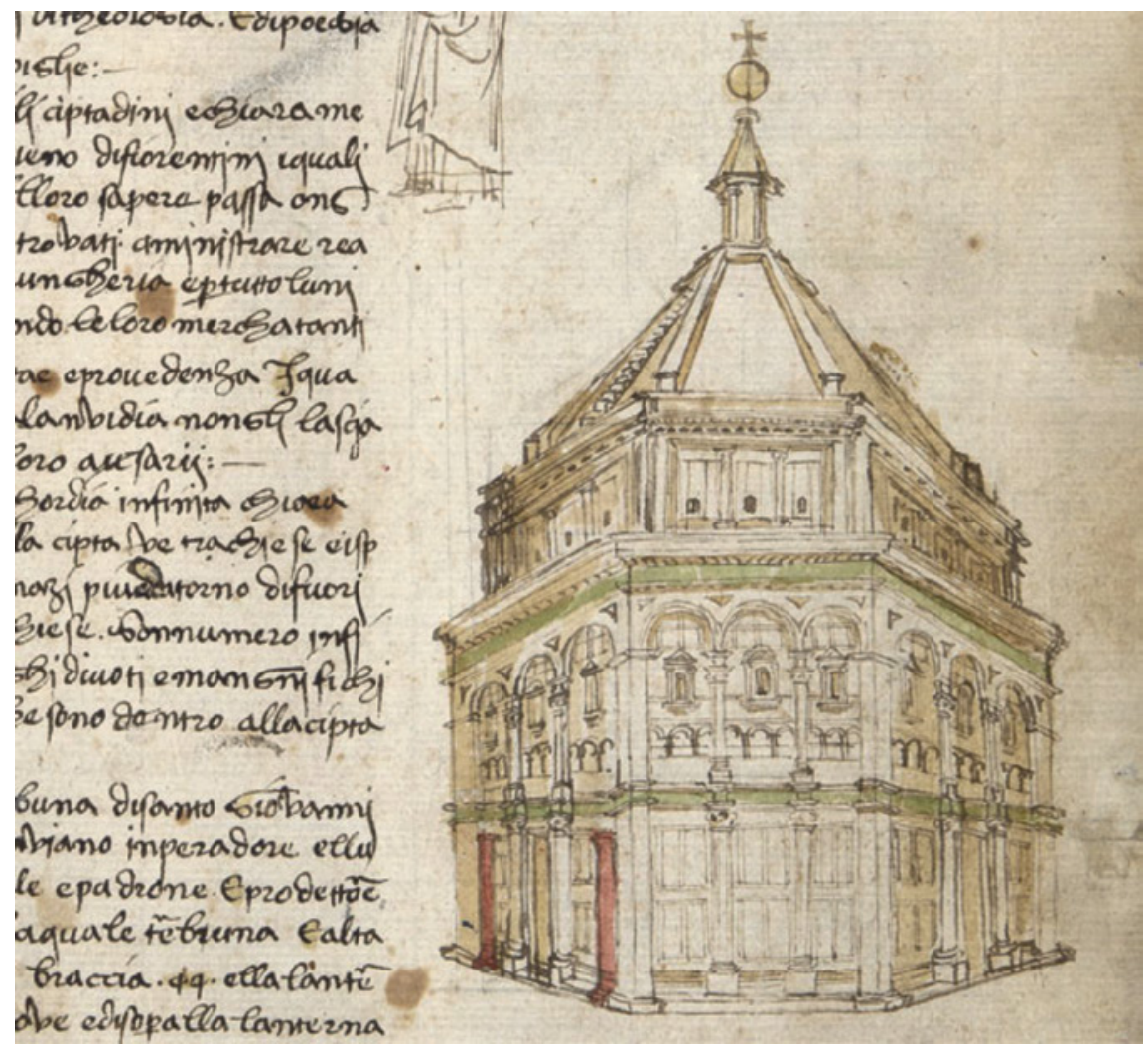

FIGURE 10.2 Marco di Bartolomeo Rustici, Veduta del Battistero, seconda metà del XV secolo; Firenze, Biblioteca del Seminario Arcivescovile Maggiore, c. 8r.

Il brano in esame, già richiamato insieme con altri da parte di Francesco Di Teodoro, rivela il ruolo primario, e allo stesso tempo diversificato, che il Battistero viene ad assumere nel pensiero di Vasari, così come nelle altrettanto articolate riflessioni sviluppate da Borghini sullo stesso monumento. Tali teorizzazioni hanno ripercussioni dirette sulle 'imprese', effimere e non solo, che nel corso del principato dei primi tre granduchi avrebbero interessato il 'Bel San Giovanni' (fig. 10.2). ${ }^{7}$

7 E. Borsook, 'Art and Politics at the Medici Court, II: the Baptism of Filippo dei Medici in 1577', Mitteilungen des Kunsthistorischen Institutes in Florenz XIII (1967), no. 1-2, 95114; E. Settesoldi, 'Gli apostoli dell'Ammannati nel Battistero di Firenze', Antichità viva XIV (1975), no. 4, 55-59; Z. Waźbiński, 'Le polemiche intorno al Battistero fiorentino nel Cinquecento', in: Filippo Brunelleschi. La sua opera e il suo tempo, Firenze 1980, vol. II, 933-950; C. Conforti, 'Feste Medicee: il battesimo, le esequie, l'apoteosi', in: M. Fagiolo (ed.), La Città effimera e l'universo artificiale del giardino: la Firenze dei Medici 
Negli apparati del 1565 per l'ingresso di Giovanna d'Austria e nel dipinto del Salone dei Cinquecento dedicato alla celebrazione della Florentia romana, il Battistero viene rappresentato come l'antico tempio di Marte, recuperando una tradizione che risale a Giovanni Villani e che, senza soluzione di continuità, col tramite degli elogia urbis rinascimentali, giunge al pieno Cinquecento (figg. 10.3-4). ${ }^{8}$ Accanto a tale interpretazione che vede il Battistero quale reliquia di Florentia, si pone l'esemplarità del Battistero come importante testimonianza dell'evoluzione artistica post-classica che trova esito nel Romanico fiorentino. ${ }^{9}$ Il duplice approccio nei confronti dell'edificio non va pertanto interpretato come una contradditoria antinomia, bensì invece come una eloquente endiade che rivela l'atteggiamento ideologicamente mutevole da parte di Vasari e di Borghini nei confronti del monumento fiorentino. Infatti, in un disegno storiografico di più ampio respiro qual è il Trattato della chiesa e vescovi fiorentini, ${ }^{10}$ lo stesso Borghini legge il Battistero come l'edificio cardine della Firenze paleocristiana e alto-medioevale. Tutto ciò emerge chiaramente da una testimonianza grafica di grande rilievo, ${ }^{11}$ appartenente allo stesso contesto cronologico della metà degli anni Sessanta del Cinquecento: nel disegno in questione viene esaltata la centralità del Battistero, ormai il cristianizzato ex-tempio di Marte, in un reticolo di edifici fiorentini civili e religiosi, romani e/o tardo antichi (fig. 10.5).

Tale importantissimo documento, delineato dalla mano di Borghini, nasce dalla lettura di più fonti, tanto di carattere antiquario, quanto frutto della

e l'Italia del '50o, Roma 1980, 101-121; A.M. Testaverde Matteini, 'La decorazione festiva e l'itinerario di "rifondazione" della città negli ingressi trionfali a Firenze tra XV e XVI secolo (II)', Mitteilungen des Kunsthistorischen Institutes in Florenz XXXIV (1990), no. 1-2, 165198; A. Fara, Bernardo Buontalenti, Milano 1995, 64-65, 139-140; G. Morolli, 'L'architettura del Battistero e "l'ordine buono antico"', in: A. Paolucci (ed.), Il Battistero di San Giovanni a Firenze, Modena 1994, 33-132.

8 Una sintesi di queste ricerche si legge in E. Ferretti, 'Da mirabilia a monumenta: Vincenzio Borghini, la memoria dell'acquedotto romano e il mito fondativo dell'origine di Firenze nelle fonti letterarie dal XIII al XVI secolo', in: Architettura e identità locali, op. cit. (nota 3), 529-551, con bibliografia. Per il disegno preparatorio della fondazione augustea di Florentia nel soffitto del Salone dei Cinquecento, E. Pillsbury, 'The Sala Grande Drawings by Vasari and His Workshop: Some Documents and New Attributions', Master Drawings XIV, no. 2 (Summer 1976), 127-146, 187-200.

9 Una bibliografia aggiornata è presente in E. Faini, Firenze nell'età romanica (1000-1211): L'espansione urbana, lo sviluppo istituzionale, il rapporto con il territorio, Firenze 2010; R. Chellini (ed.), Chronica de Origine Civitatis Florentiae, Roma 2009, $75^{-81 .}$

10 Vincenzio Borghini, Discorsi, Firenze, Giunti, 1585, II, 337-598. Per questo testo: M. Fubini Leuzzi, 'Una introduzione per la lettura storica dei Ricordi di Vincenzio Borghini', in: I Ricordi, op. cit. (nota 2), 13-100: 8o-86.

11 E. Carrara, 'Scheda 2.2: Miscellanea di varia erudizione antiquaria (spogli sulle famiglie fiorentine)', in: G. Belloni, R. Drusi (eds.), Vincenzio Borghini. Filologia e invenzione nella Firenze di Cosimo I, Firenze 2002, 16-20: 16. 


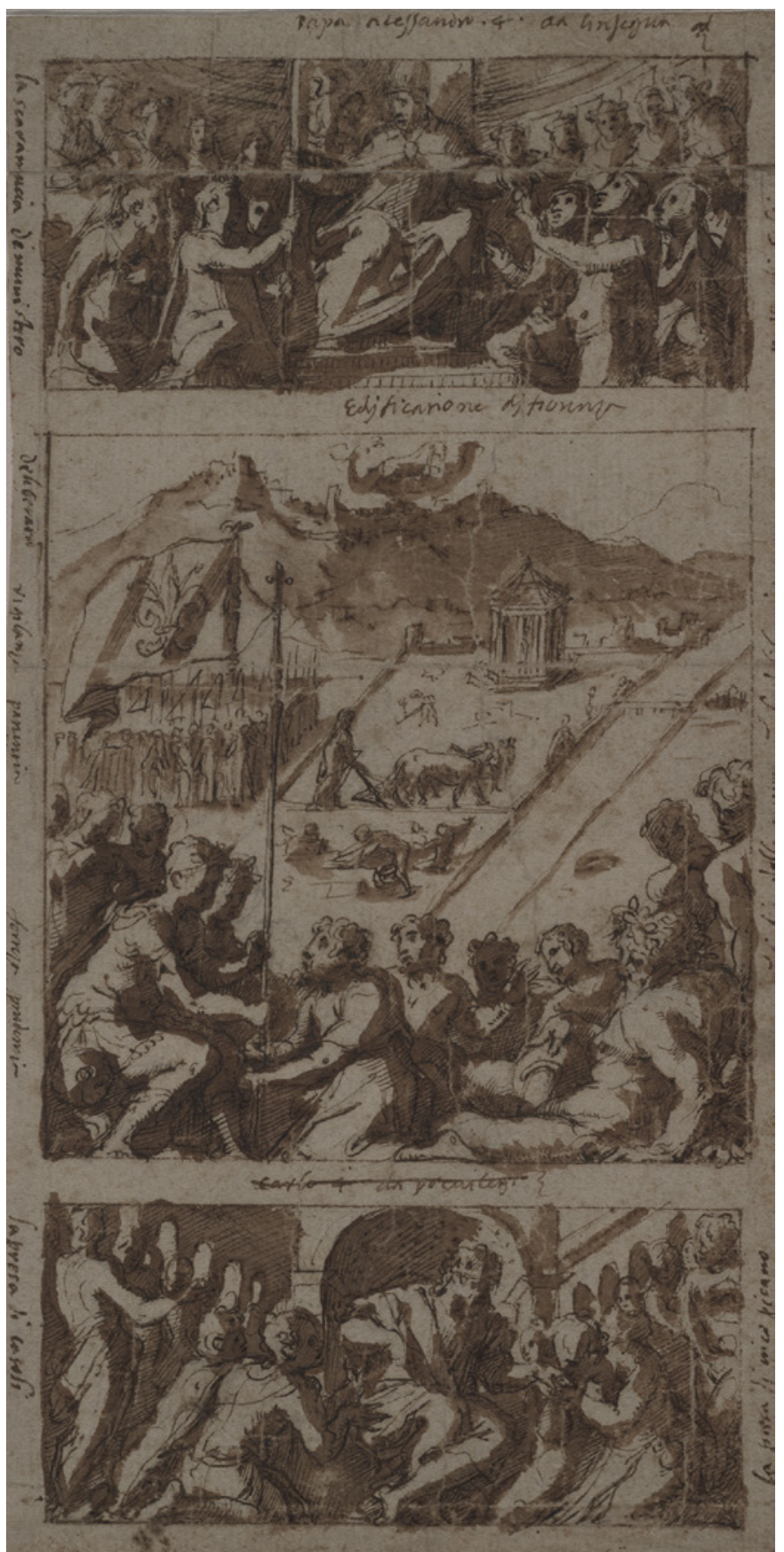

FIGURE 10.3 Giorgio Vasari, Disegno preparatorio per il riquadro dedicato alla fondazione di Florentia, ca. 1563, Cambridge, Harvard University, Fogg Art Museum, 1932.157.B. 


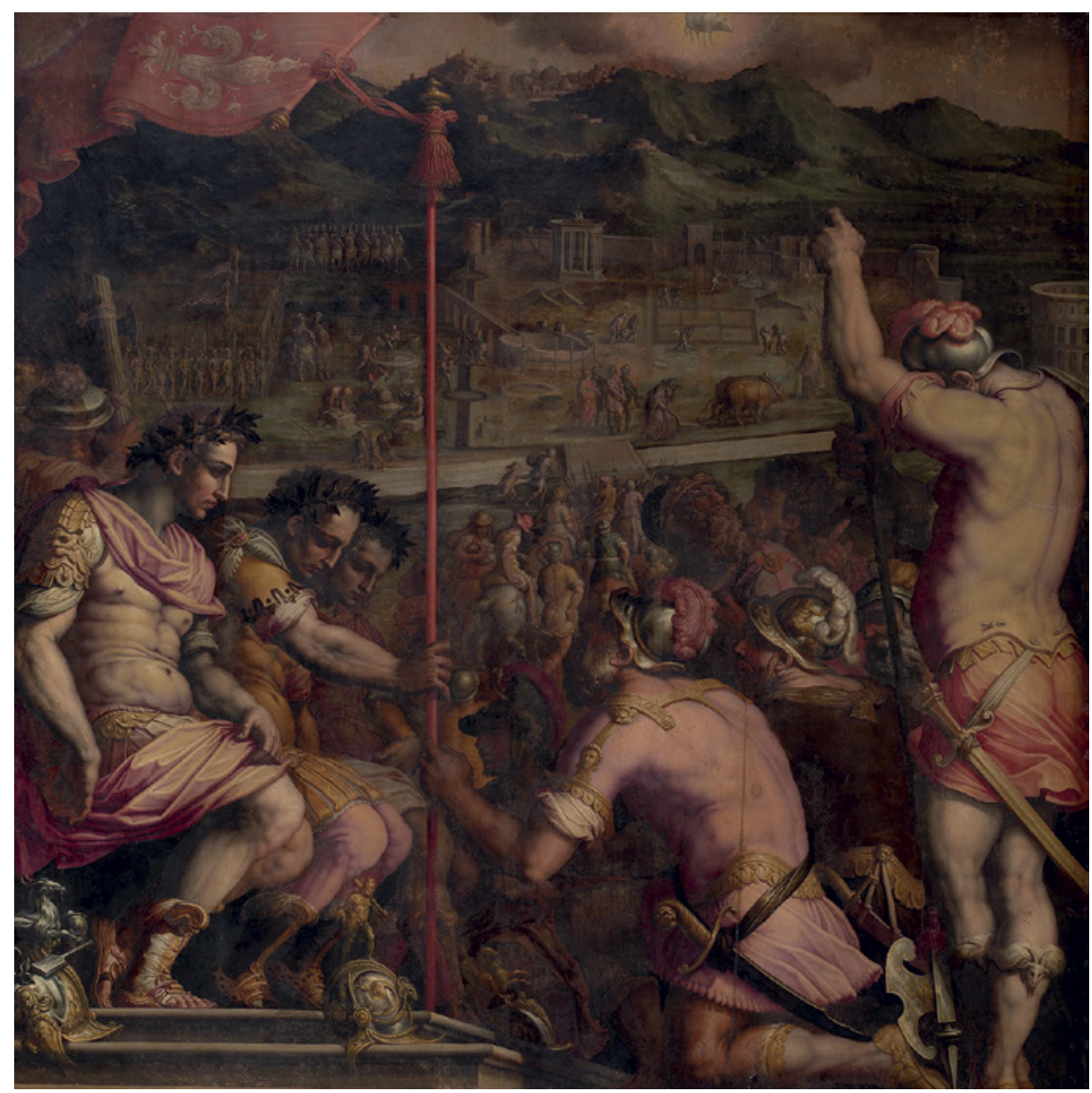

FIG URE 10.4 Giorgio Vasari, La fondazione di Florentia, 1565, Firenze, Palazzo Vecchio, Salone dei Cinquecento FOTO: MUSEI CIVICI FIORENTINI

tradizione storica e memorialistica cittadina. In particolare, appaiono pienamente evidenziati per la prima volta i punti di contatto con la Chronica de origine civitatis Florentiae. ${ }^{12}$ Come ha osservato Davis, ${ }^{13}$ questa rilevante fonte anonima del XIII secolo, che dovette essere presente nel grande laboratorio di indagini storiche approntato dal benedettino, ${ }^{14}$ restituisce l'immagine della Firenze altomedioevale come speculare riproposizione della Roma

\footnotetext{
12 Chellini, Chronica de Origine, op.cit (nota 9); J.R. Woodhouse, 'Vincenzio Borghini's View of Charlemagne's Empire: A Study with Unpublished Texts', Viator XIX (1988), 355-376.

13 C.T. Davis, 'Topographical and Historical Propaganda in Early Florentine Chronicles and in Villani', Medioevo e Rinascimento II (1988), 33-51: 37-38.

14 R. Drusi, 'Scheda 5.1.2, Giovanni Villani nell'edizione giuntina postillata dal Borghini (59Borgh)', in: Vincenzio Borghini. Filologia e invenzione, op. cit. (nota 11), 178-182.
} 


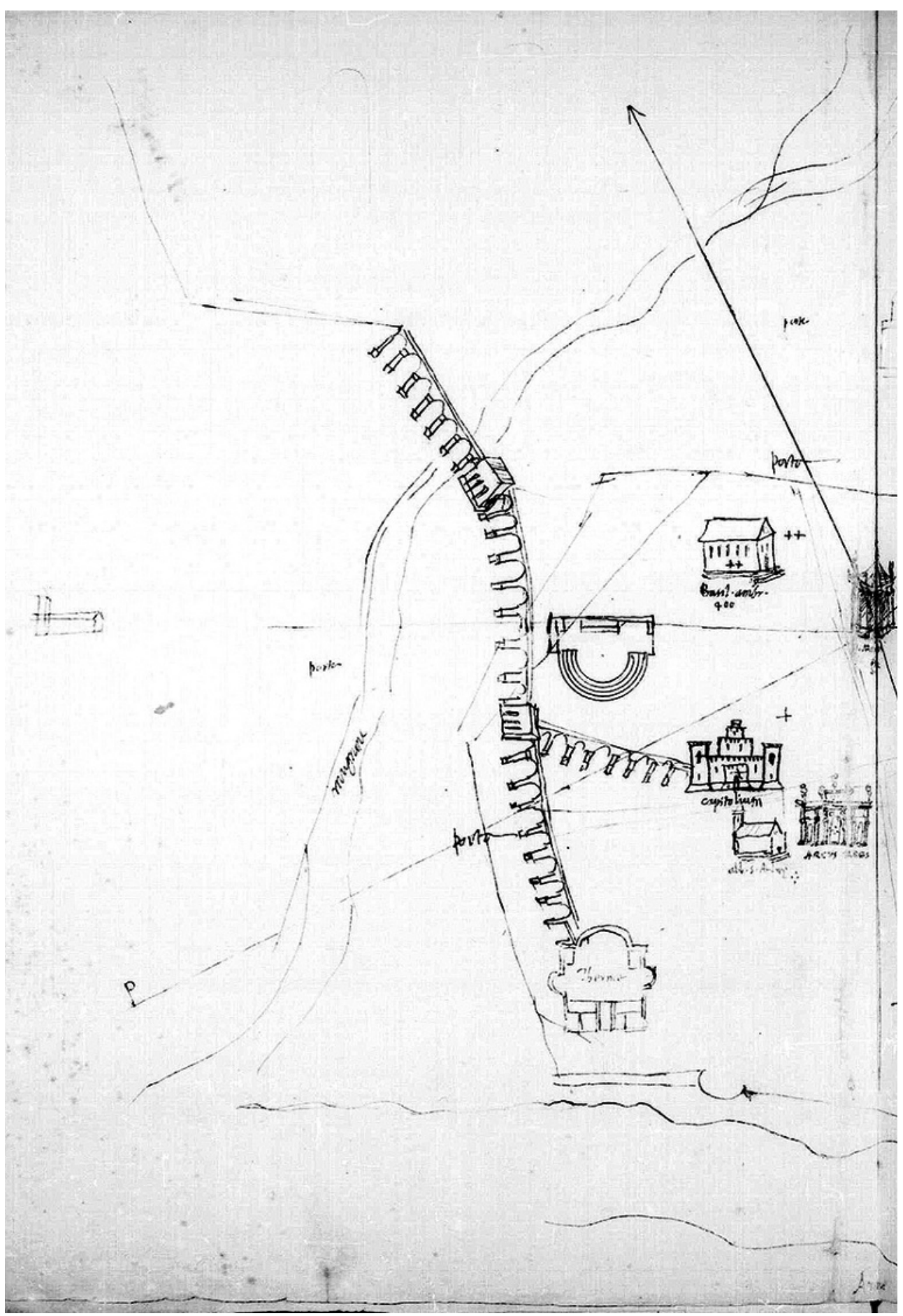

FIGURE 10.5 Vincenzio Borghini, Ricostruzione di Florentia romana e paleocristiana, ca. 1570; BNCF, Ms. Magliabechiano, XXV, 551, cc. 26v.-27r. 


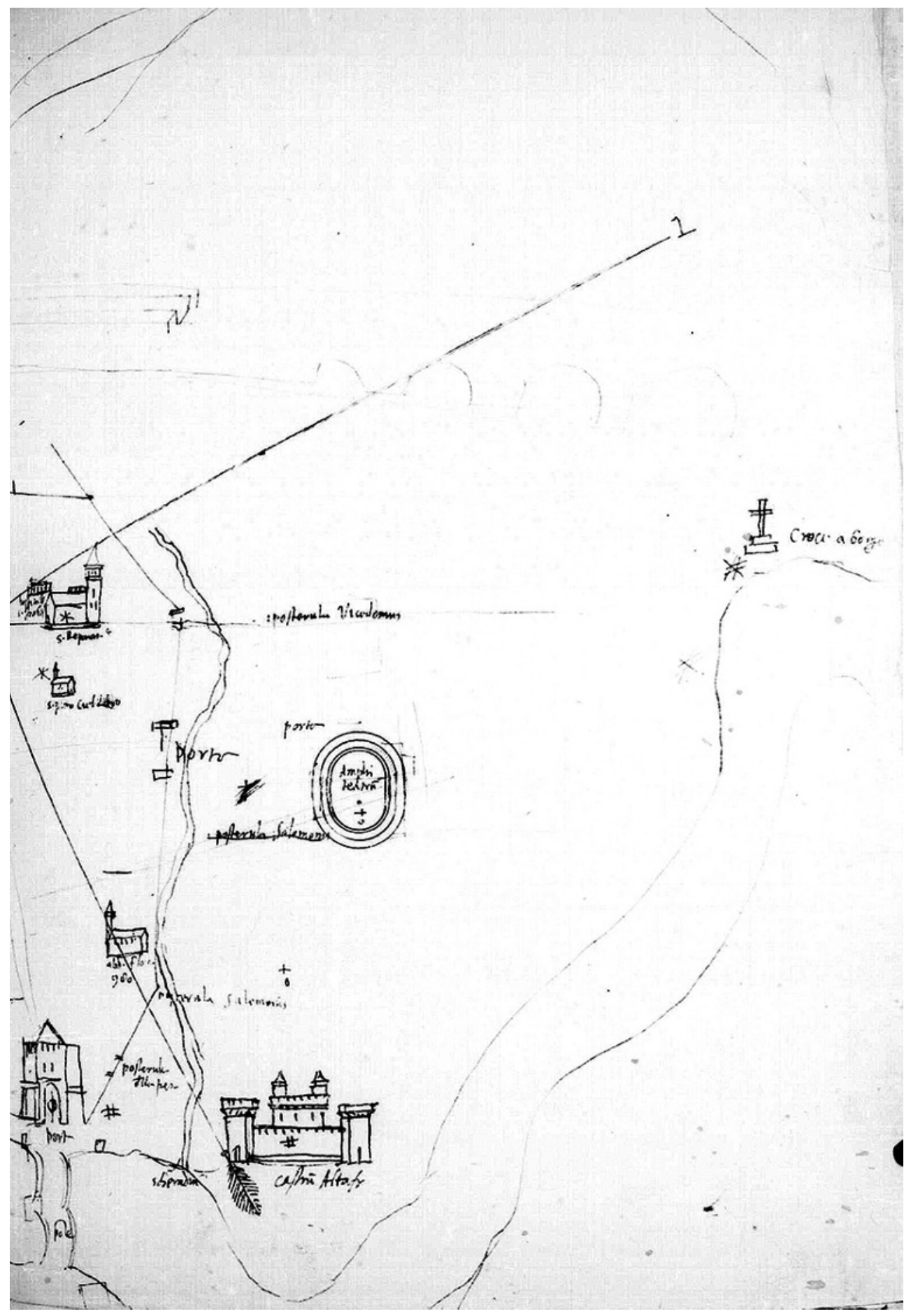


paleocristiana proprio attraverso le sue chiese più importanti, con una particolare attenzione al parallelismo fra il San Giovanni Battista fiorentino e il battistero di San Giovanni in Laterano. Al centro della pianta si vede il Battistero, circondato da una serie di edifici sacri, presentati come paleocristiani, che completano la struttura urbana dominata dai monumenti della Florentia romana, richiamati con continuità dalle ricostruzioni mitografiche medioevali e umanistiche: il Campidoglio, l'acquedotto, le mura, le terme etc. La città evocata da Borghini non è più sotto il segno di Marte, ma sotto il segno di S. Giovanni Battista: gli assi cardinali sono generati dal Battistero, che viene presentato come il centro ideale della città. ${ }^{15}$

\subsection{Dal tempio di Marte al 'bel San Giovanni'}

La tradizione medioevale aveva tramandato che nella ricostruzione di Firenze, dopo la distruzione di Totila, il modello era stato nuovamente Roma: la Roma costantiniana, la Roma cristiana. Borghini, tuttavia, cerca di uscire dalla dimensione del mito e trasformare la leggenda in storia, a partire proprio dal Battistero. L'edificio sacro, omphalos urbano, diviene il più spettacolare collegamento fra le due città: la rilevanza religiosa del Battistero Lateranense, infatti, si rispecchia nella centralità del Battistero fiorentino, umbilicus urbis della Florentia cristiana, precorritrice della Firenze medicea devota e profondamente religiosa. ${ }^{16}$ Nella restituzione della compagine urbana tardo-antica spicca, inoltre, la medicea chiesa di S. Lorenzo che, quale edificio di culto di origine paleocristiano, ${ }^{17}$ è un altro tassello significativo della narrazione della storia fiorentina del Cristianesimo delle origini tracciata da Borghini: la 'basilica ambrosiana', cioè la 'chiesa vostra di S. Lorenzo' - come scriveva Borghini a Francesco I l'8 giugno 1578 - è tanto più importante perché legata a

15 Ferretti, 'Da mirabilia a monumenta, op. cit. (nota 8), 533-555.

16 A. D'Addario, 'Testimonianze archivistiche, cronachistiche e bibliografiche', in: La comunità cristiana fiorentina e toscana nella dialettica religiosa del Cinquecento, Florence 1980, 23-194: 86; M. Cavarzere, 'Cosimo I pater ecclesiae, tra eresia, riforma religiosa e ragion di Stato', in: Cosimo I de' Medici: itinerari di ricerca tra arte, cultura e politica, Annali di storia di Firenze IX (2014), 77-86.

17 E. Carrara, 'Scheda 2.4, Borghini: Memorie e notizie d'antichità diverse', in: Vincenzio Borghini, Filologia e invenzione, op. cit. (nota 11), 25-30: 26; E. Ferretti, 'Sacred Space and Architecture in the Patronage of the First Grand Duke of Tuscany: Cosimo I, San Lorenzo and the Consolidation of the Medici Dynasty', in: R. Gaston, L. A. Waldman (eds.), San Lorenzo. A Florentine Church (Villa I Tatti Series, 33), Florence/Cambridge (MA) 2017, 504-524. 


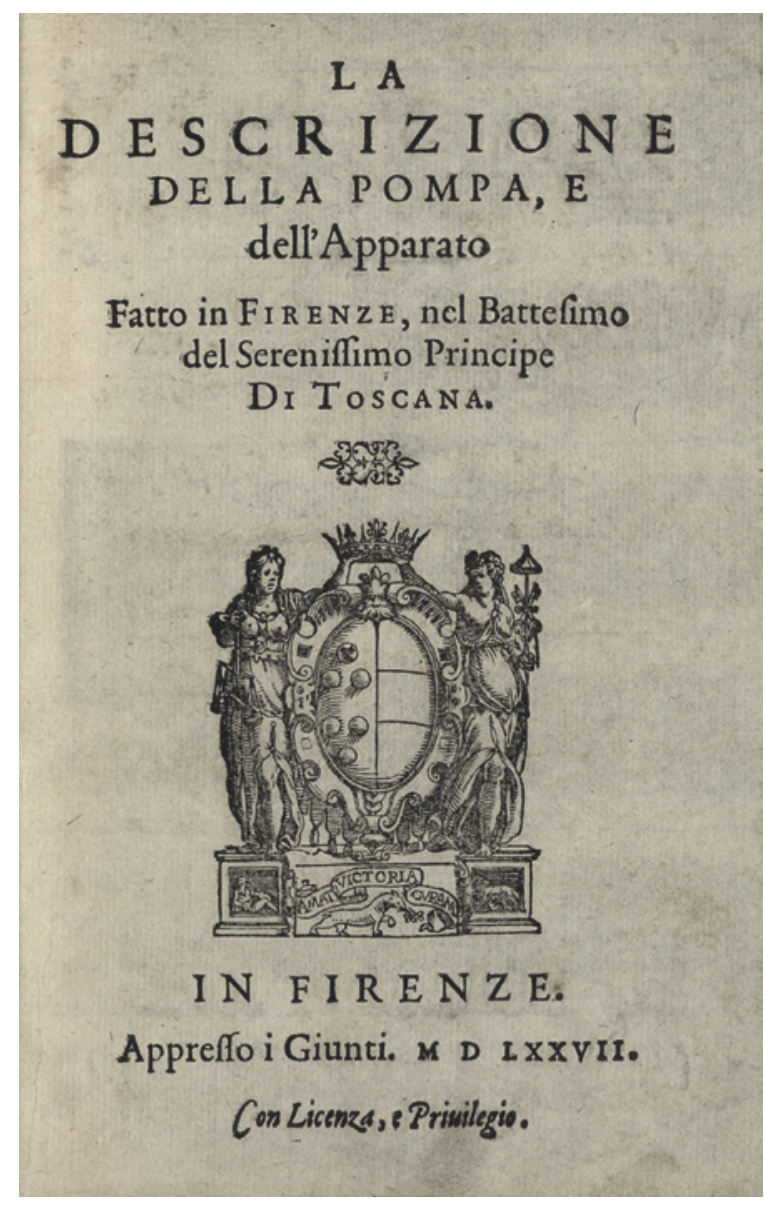

FIGURE 10.6

Frontespizio della

Descrizione della Pompa, $e$ dell'Apparato fatto in Firenze, nel Battesimo del Serenissimo Principe di Toscana, Firenze, Giunti, 1577

S. Ambrogio, di cui 'a Roma si stampano di nuovo l'opere sue'. ${ }^{18}$ All'anno precedente, ossia il 1577, risale l'edizione della Descrizione della Pompa, e dell'Apparato fatto in Firenze, nel Battesimo del Serenissimo Principe di Toscana curata da Borghini (fig. 10.6), dove si legge:

[...] prima che ad altro vegniamo, è da sapere che la forma del Battesimo o vero chiesa, dove si è fatto questo battesimo, la quale fu già Tempio di Marte Vendicatore, è quasi la medesima che 'l Panteon di Roma; salvo che dove quella è di figura tonda, onde piglia il suo nome, questa è

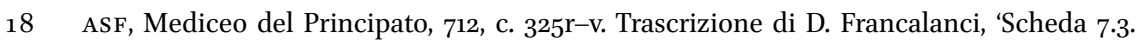
Borghini a Francesco I de' Medici per la chiesa di San Lorenzo', in: Vincenzio Borghini, Filologia e invenzione, op. cit. (nota 11), 376-379: 378. 
ottangulare, et ha negl'angoli alcuni pilastri, i quali tornano dentro come colonne quadre corinthie. E fra l'una e l'altra, divise in ugual spazio, sono due colonne del granito dell'Elba, del quale tante se ne veggiono in Roma, del medesimo ordine. E così vengono a restare in ciascuno degl'otto lati tre spazii, che sarebbono in tutto ventiquattro. Ma perché dalla banda di ponente furono levate già le due colonne che vi erano, e gettato un arco per fare la Cappella Maggiore et accomodare da potervi celebrare i sacri misterii et ufizii, secondo l'uso delle chiese christiane, et altre tre // ne occupano le tre porte principali [...] vengono a restare gli spazii da potersene servire solamente diciotto. ${ }^{19}$

L'ecfrasis borghiniana è la versione letteraria delle informazioni visive ricavabili dai rilievi commissionati dall'erudito benedettino a uno dei suoi più fidati collaboratori, Giovanni Battista Naldini (1535-1591), nell'autunno del 1575 (figg. 10.7-8). ${ }^{20}$ Inoltre, questi stessi elaborati furono la base per realizzare le incisioni che funsero da illustrazione per i Discorsi (fig. 10.9), opera postuma pubblicata a Firenze tra il 1584-1585 e dedicata a Francesco I de' Medici dove, a proposito del Battistero, si legge:

[...] La ragione poi delle parti di dentro era diversa, e come il Panteon è tutto uniforme, così era questo nostro in tutte le sue otto facce ordinato alla medesima guisa colle due colonne, che nelle sette altre facce che si veggono oggi; ma l'ottava, ove era l'entrata, è la parte che secondo coloro (che non errano in questo, purché siano intesi) fu guasta: perché tolte vie le colonne, scarnata la grossezza del vestibulo, rimurata la porta e rotto l'architrave piano, fu girato quell'arco che oggi vi si vede e vi sono rimasi anchora i capitelli e parte de' pilastri, che come nell'altre facce v'erano, e sono né più né meno rotti che si veggan quegli della cappella di testa del Panteon, e par quasi che e' siano a posta rimasi per testimoni, che ella havea la medesima forma che l'altre [...] E // di qui nacque l'occasione che fusse così capovolto San Giovanni; se ella havesse oltre a questo el portico, come ha oggi il Panteon, che per alcuno accidente sia venuto manco, sarebbe uno indovinare, e se si cavasse ma in quel luogo si potrebbe forse da quel che si trovasse di sotto di fondamenti e d'altro darne giudizio. ${ }^{21}$

19 La descrizione della Pompa, e dell'Apparato fatto in Firenze, nel Battesimo del Serenissimo Principe di Toscana, Firenze, Giunti, 1577, 5-6.

20 BNCF, Cod. Magl., 551, c.167r. E. Carrara, 'Su alcuni disegni di Naldini presenti in un manoscritto di Vincenzio Borghini', Fontes VI, 11-12, (2003 [2006]), 135-155: 138-139.

21 Vincenzio Borghini, 'Dell'origine della città di Firenze', in: Discorsi, op. cit. (nota 10), I, $1-206,164-165$. 


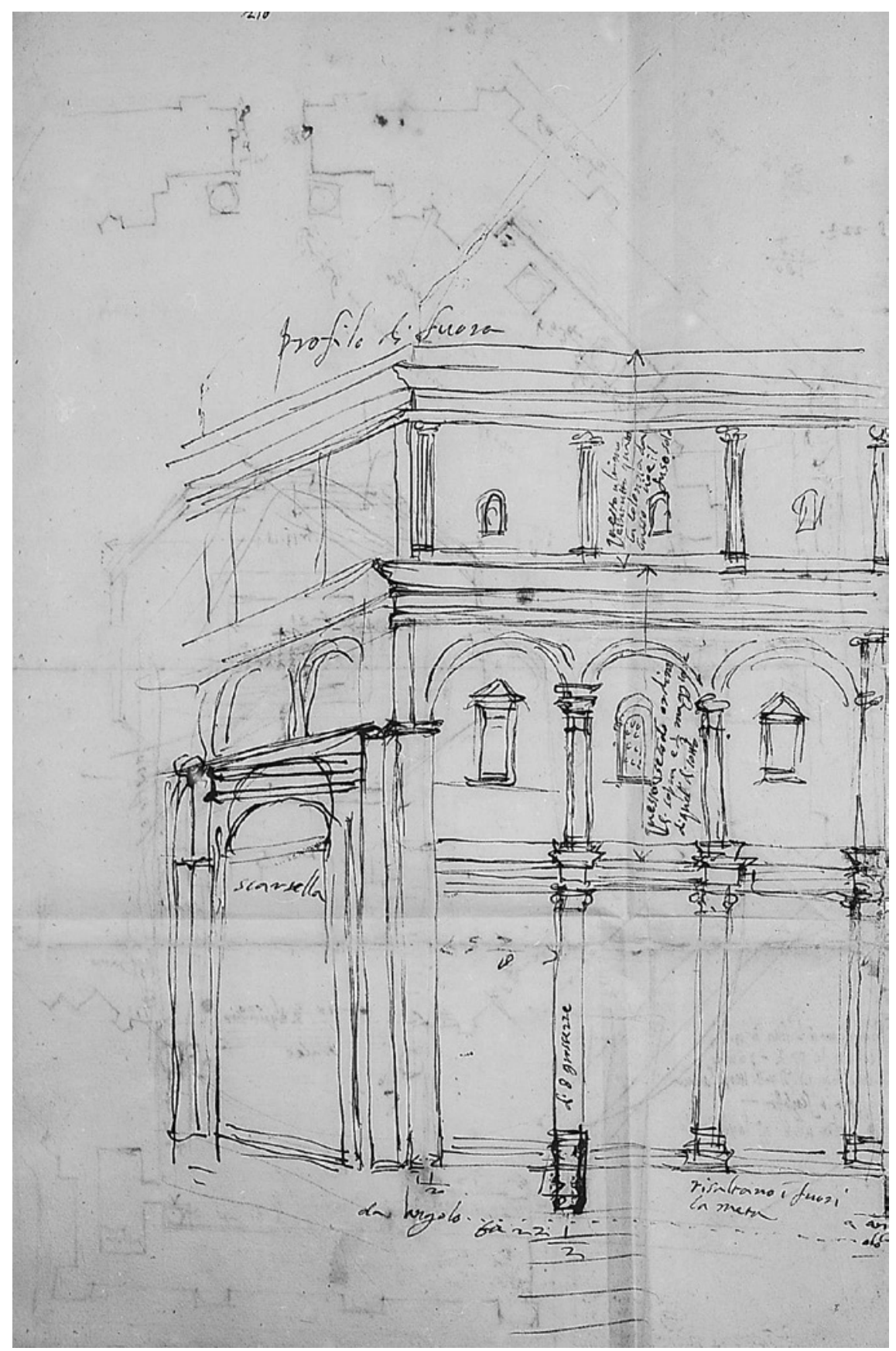

FIGURE 10.7 Giovanni Battista Naldini, Rilievi del Battistero di Firenze; BNCF, Ms. Magliabechiano, XXV, 551, c. 216r 


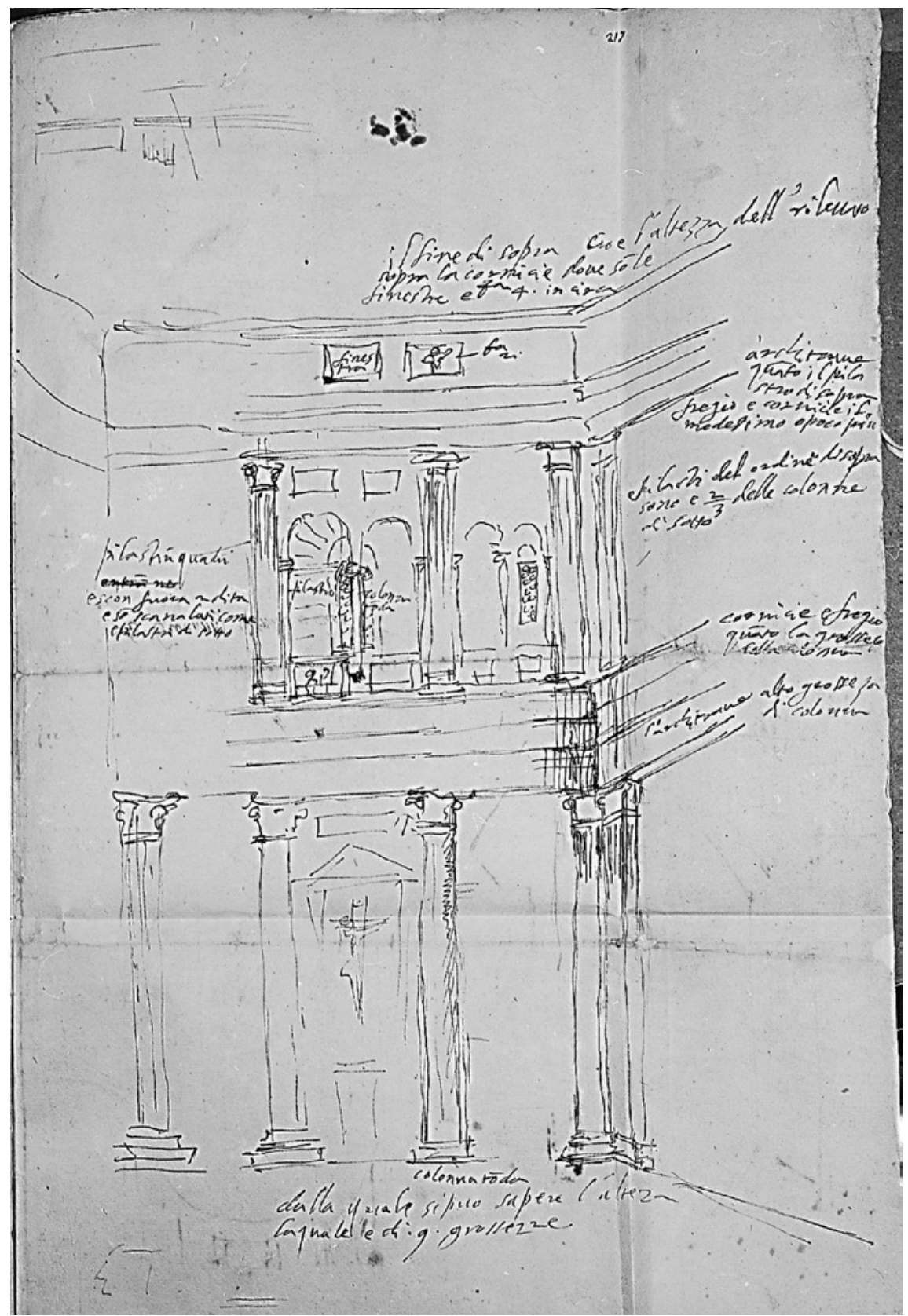

FIGURE 10.8 Giovanni Battista Naldini, Rilievi del Battistero di Firenze; BNCF, Ms. Magliabechiano, XXV, 551, c. 217r 


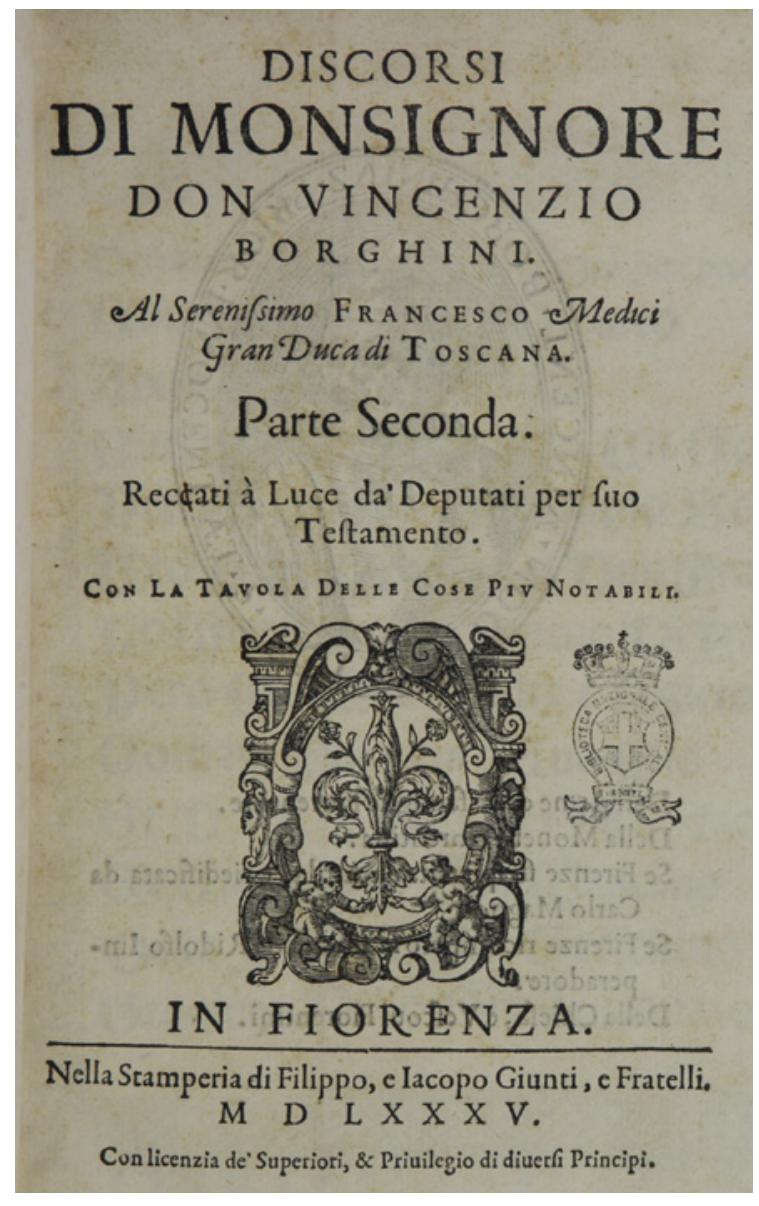

FIGURE 10.9

Vincenzio Borghini, Discorsi, Firenze, Giunti, 1584-1585, II, frontespizio

Le incisioni dedicate al Battistero (figg. 10.10-10.11) che compaiono nei Discorsi - precedute da uno studio attento del monumento che si fonda su un puntuale rilievo eseguito da Naldini e da disegni interpretativi dello stesso Borghini (figg. 10.12-10.13) - mostrano dunque una versione del Battistero intersecata con il modello del Pantheon che, come è noto, è stato cristianizzato sul principio del viı secolo: si nota il frontone triangolare, la copertura in parte gradonata, e una sorta di pronao esemplato nella sua articolazione planimetrica del monumento antico. ${ }^{22}$ Lo stesso vale per l'interno, con le grandi edicole

22 L'importanza del modello dell'edificio romano nell'architettura quattro-cinquecentesca fiorentina è sottolineata da Vasari in più occasioni, fra cui spicca per rilevanza il passo nella Vita di Brunelleschi (Vasari, Le vite, op. cit. (nota 4), III, 135-198: 153) e soprattutto, all'interno della biografia di Andrea Sansovino: 'Vi fece dunque di macigno un componimento 

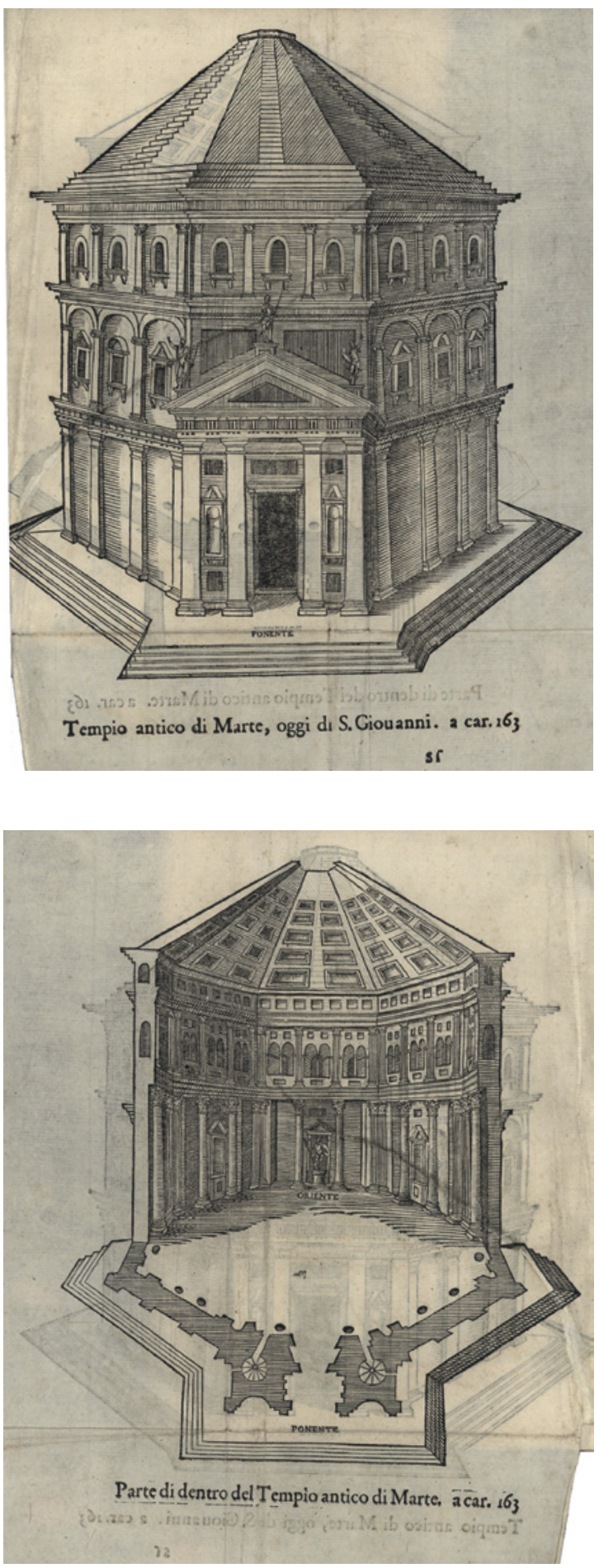

FIGURE 10.10-10.11

Veduta esterna ed interna del Battistero fiorentino, da Vincenzio Borghini, Discorsi, 1584-1585. Incisioni 

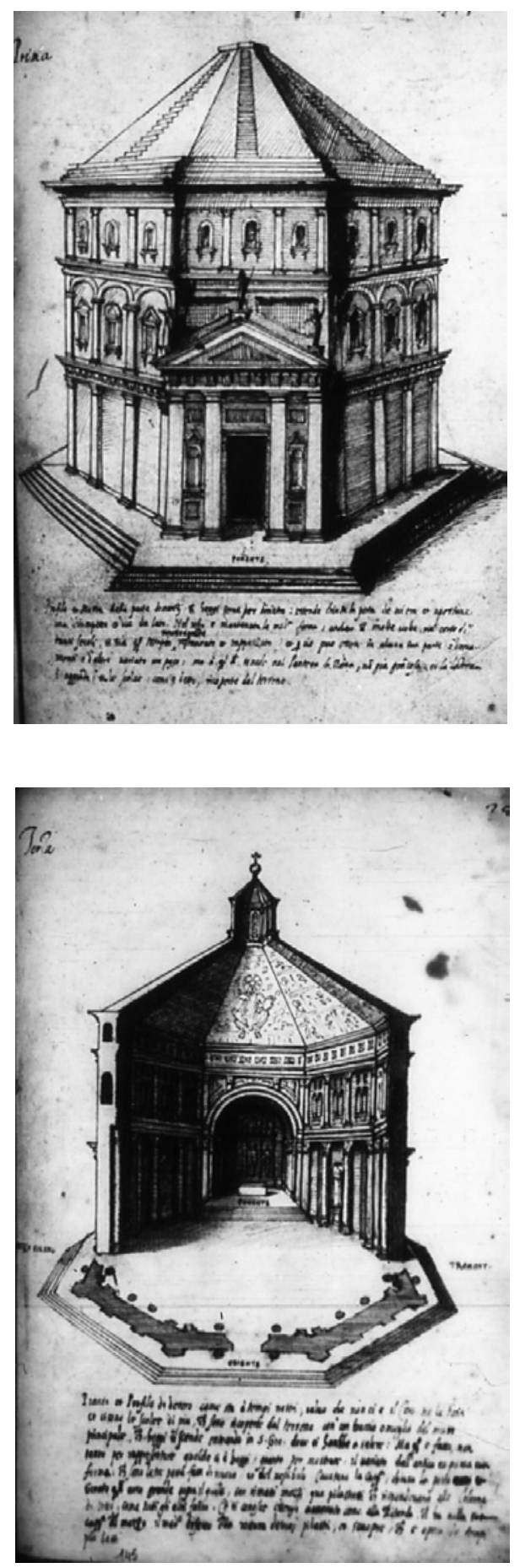

FIGURE 10.12

Vincenzio Borghini, Ricostruzione del Battistero di Firenze come tempio antico; BNCF, Ms. Magliabechiano, XXV, 545, c. $134 \mathrm{r}$
FIGURE 10.13

Vincenzio Borghini, Ricostruzione del Battistero di Firenze come tempio antico; BNCF, Ms. Magliabechiano, XXV, 545, C. $145 \mathrm{r}$ 
che richiamano gli analoghi elementi del tempio romano, riproposti, non a caso, concettualmente anche negli allestimenti per il Battesimo del $1577 \cdot{ }^{23}$

Le considerazioni di Borghini sul Battistero rientrano nelle ricerche alla base del già citato Trattato della chiesa e vescovi fiorentini. ${ }^{24}$ Nell'ambito di questo ulteriore lavoro, Borghini ripercorre non solo le fonti d'archivio e i testi classici e patristici: il suo interesse, infatti, si appunta anche sull'architettura degli edifici chiesastici antichi esistenti a Firenze. Le considerazioni che Borghini elabora sulle chiese paleocristiane sono state studiate con attenzione da Christoph Jobst, ${ }^{25}$ che le ha messe in relazione con le coeve teorizzazioni di Onofrio Panvinio (1530-1568) e Cesare Baronio (1538-16o7) ${ }^{26}$ Borghini cercò

d'ordine corinto, con dodici colonne tonde, cioè sei da ogni banda; e sopra le colonne posto l'architrave, fregio e cornice, fece una volta a botte, tutta della medesima pietra, con uno spartimento pieno d'intagli, che fu cosa nuova, varia, ricca e molto lodata. Ben è vero che se il detto spartimento della volta fusse ne' diritti delle colonne venuto a cascare con le cornici, che vanno facendo divisione intorno ai quadri e tondi che ornano quello spartimento, con più giusta misura e proporzione, questa opera sarebbe in tutte le parti perfettissima, e sarebbe stato cosa agevole il ciò fare. Ma secondo che io già intesi da certi vecchi amici d'Andrea, egli si difendeva con dire d'avere osservato nella volta il modo del partimento della Ritonda di Roma, dove le costole, che si partono dal tondo del mezzo di sopra, cioè dove ha il lume quel tempio, fanno dall'una all'altra i quadri degli sfondati dei rosoni che a poco a poco diminuiscono; et il medesimo fa la costola, perché non casca in su la dirittura delle colonne. Aggiugneva Andrea, se chi fece quel tempio della Ritonda - che è il meglio inteso e misurato che sia e fatto con più proporzione - non tenne di ciò conto in una volta di maggior grandezza e di tanta importanza, molto meno dovea tenerne egli in uno spartimento di sfondati minori. Nondimeno molti artefici, e particolarmente Michelagnolo Buonaroti, sono stati d'openione che la Ritonda fusse fatta da tre architetti, e che il primo la conducesse al fine della cornice che è sopra le colonne; l'altro dalla cornice in su, dove sono quelle finestre d'opera più gentile, perché invero questa seconda parte è di maniera varia e diversa dalla parte di sotto, essendo state seguitate le volte senza ubidire ai diritti con lo spartimento; il terzo si crede che facesse quel portico che fu cosa rarissima. Per le quali cagioni i maestri che oggi fanno questa arte non cascherebbono in così fatto errore, per iscusarsi poi come faceva Andrea'. (Vasari, Le vite, op. cit. (nota 4), IV, 270-289: 273-274). Si noti che il brano è presente solo nella seconda edizione.

23 Vedi più supra nota 7.

24 Vedi più supra nota 10.

25 Chr. Jobst, 'Die christliche Basilika. Zur Diskussion eines Sakralbautypus in italienischen Quellen der posttridentinischen Zeit', Zeitsprünge. Forschungen zur Frühen Neuzeit I (1997) no. 3-4, 698-749: 701-733.

26 M.G. Turco, 'Cesare Baronio e i dettami tridentini nelle sistemazioni presbiteriali romane', in: P. Tosini (ed.), Arte e committenza nel Lazio nell'età di Cesare Baronio, atti del convegno internazionale di studi (Frosinone-Sora, 16-18 maggio 2007), Roma 2009, 87-107; L. Spera, 'Cesare Baronio, "peritissimus antiquitatis" e le origini dell'archeologia cristiana', in: G.A. Guazzelli, R. Michetti, F. Scorza Barcellona (eds.), Cesare Baronio tra santità e scrittura storica, atti del colloquio internazionale di studi (Roma 2007), Roma 2012, 393-423. Per Panvinio, vedi infra nota 28. 
di dare una forma coerente e sistematica alla propria riflessione su questi temi, giungendo a ciò che Jobs definisce 'teoria della conversione': il dotto benedettino, infatti, riteneva che la basilica cristiana fosse una filiazione diretta della basilica forense, ovvero la versione cristianizzata di tale tipologia pagana; e, pertanto, proprio negli antichi edifici chiesastici fiorentini trovava uno snodo concettuale fondamentale nello sviluppo del proprio ragionamento. Secondo Jobst, la sua interpretazione della basilica e quel concetto di forte continuità fra l'edificio pagano e quello di culto paleocristiano appaiono essere il frutto di una visione marcatamente di carattere antiquario, finalizzata alla dimostrazione dell'origine romana della città Firenze. Secondo questa visione, le chiese fiorentine sarebbero prima di tutto testimonianze della sopravvivenza dell'antica basilica forense. Il Battistero, in questo senso, è portatore di valenze ancora più cogenti rispetto a San Lorenzo e o San Pier Scheraggio, per essere già stato riconosciuto e studiato nelle sue qualità fisiche dagli architetti del Rinascimento come edificio antico (fig. 10.14), specifico emblema - come ha scritto Amedeo Belluzzi - di una versione autoctona del classicismo. ${ }^{27}$ In questo modo le ricerche borghiniane andavano a saldarsi con quella 'archeologia cristiana' che aveva trovato come straordinario primo cultore il già menzionato Onofrio Panvinio ${ }^{28} \mathrm{e}$ che troverà un'ulteriore esito con la Roma sotterannea di Antonio Bosio, opera postuma nel $1632 .{ }^{29}$

27 A. Belluzzi, Giuliano da Sangallo e la chiesa della Madonna dell'Umiltà a Pistoia, Firenze 1993, 62; T. Buddensieg, 'Criticism and Praise of the Pantheon in the Middle Ages and the Renaissance', in: R.R. Bolgar (ed.), Classical Influences on European Culture A.D. 500-1500: Proceedings of an International Conference Held at Kings College, Cambridge, April 1969, Cambridge 1971, 259-267; A. Nesselrath, 'Impressions of the Pantheon in the Renaissance', in: T.A. Marder, M. Wilson Jones (eds.), The Pantheon. From Antiquity to the Present, Cambridge 2015, 255-295.

28 K.A. Gerbsach, 'Onofrio Panvinio OSA, and His Florentine Correspondents Vincenzio Borghini, OSB, Pietro Vettori, Francesco de' Medici', Analecta Augustiniana LX (1997), 207-279. In termini generali, si veda da ultimo, S. Bauer, 'Panvinio Onofrio', Dizionario Biografico degli Italiani, LXXXI (2014), 36-39.

29 G. Cantino-Wataghin, 'Archeologia e "archeologie". Il rapporto con l'antico fra mito, arte e ricerca', in: S. Settis (ed.), Memoria dell'antico nell'arte italiana. I. L'uso dei classici, Torino 1984, 169-217; I. Herklotz, 'Antonio Bosio e Giovanni Severano. Precisazioni su una collaborazione', Studi romani LVI, 1-4 (2008), 233-248; I. Herklotz, 'Antonio Bosio e Carlo Bascapé: ricerca di reliquie e studio delle catacombe nel XVII secolo', in: Idem, Apes urbanae. Eruditi, mecenati e artisti nella Roma del Seicento, Città di Castello 2017, 49-70. 


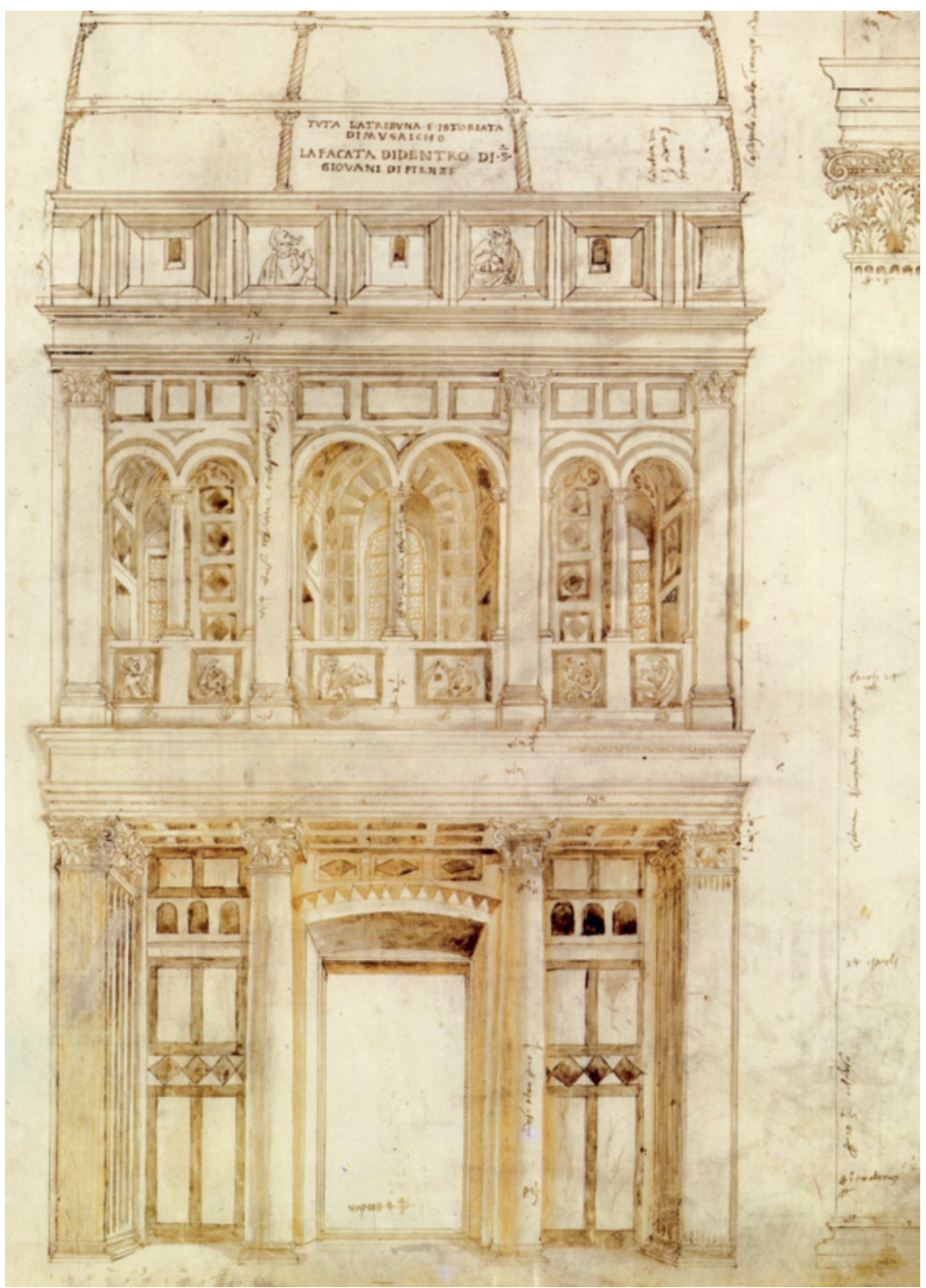

FIGURE 10.14 Giuliano da Sangallo, Il Battistero di San Giovanni a Firenze, inizio del XVI secolo; BAV, Codice Barberiniano Latino 4424, c. 34r 